\title{
Cytogenetical and agronomical aspects of radiation induced marker trait mutants in sesame (Sesamum indicum $\mathrm{L}$.)
}

\author{
Sandipan Chowdhury, Animesh K. Datta and Susmita Maity \\ Department of Botany, Genetics and Plant Breeding Section, University of Kalyani, Kalyani- 741235, India \\ dattaanimesh@gmail.com
}

Abstract: Morphological mutants in sesame (Sesamum indicum L.; Family : Pedaliaceae) with distinctive marker traits namely leaf of narrow type, elongated, thick, ovate, ternate with long petiole and with white and pigmented flowers induced following X-ray and gamma-ray irradiations showed monogenic recessive inheritance. Control and mutants had $2 n=26$ chromosomes always and the chromosomes formed bivalent (control: $12.93 /$ cell, mutant: 12.26 /cell to $12.93 /$ cell) and univalents (control: $0.14 /$ cell; mutant $0.14 /$ cell to $1.48 /$ cell) which tended to form variable groups ( 3 to 10 ) in $46.81 \%$ to $65.20 \%$ meiocytes (8 group class being frequent). Univalent frequency/cell was relatively higher in long petiole (1.48/cell). Predominant chromosomal association noted among the plant types was 13 II (control: 92.96\%; mutants: $50.00 \%$ to $95.88 \%$ ). Anaphase I segregation of chromosome was mostly equal $(13 / 13)$ in the plant types (control: $100.0 \%$, mutant: $98.80 \%-100.00 \%$ ). Pollen fertility was $83.50 \%$ in control and varied from $38.90 \%$ to $80.20 \%$ in the mutants. Pollen fertility and A1 chromosome separation was non-correlated. Analysis of quantitative parameters at $\mathrm{M}_{4}$ (true breeding plants were assessed by RBD with three replications each) revealed that most of the mutants (thick leaf, narrow leaf, elongated leaff were beneficial for their direct selection, while the others may be exploited in cross breeding programme.

Keywords: Sesame, mutants, marker traits, cytogenetics and agronomical features.

Introduction

Improvement of crop plants through induction of mutation has been emphasized (Gaul, 1964; Brock, 1977; Datta \& Biswas, 1985; Kharkwal, 2000; Datta \& Rang, 2001; Mukherjee \& Datta, 2006; Iqbal \& Datta, 2007). A research programme on radiation induced mutagenesis has been initiated to induce genetic variations and to screen desirable 'plant type' mutations for efficient plant breeding in Sesame (Sesamum indicum L.; Family: Pedaliaceae), an oil yielding plant of commerce and 20 viable morphological mutants are screened (Chowdhury \& Datta, 2008). This communication reports on the cytogenetical and agronomical aspects of some morphological marker mutants (related to leaf and pollen) induced following different doses of $\mathrm{x}$-ray and gammaray irradiations. Marker traits are always useful in genetics and breeding as they are easily scorable and selectable in field condition.

Materials and Methods

Dry and filled seeds (moisture content: 9.56\%) of Sesamum indicum $\mathrm{L}$. var. B-67 (obtained from Pulses and Oil Seed Research station, Berhampur, West Bengal;) highly adaptive to plains of West Bengal (Sengupta \& Chatterjee, 1982) were treated with gamma-rays (doses :
$50,100,200$ and $300 \mathrm{~Gy}$; source ${ }^{60} \mathrm{Co}$ at the rate of 1.3 Gy per minute, irradiation at Saha Institute of Nuclear Physics, Salt Lake, Kolkata, West Bengal) and $X$ - rays (doses: 50, 100, 200 and 300 Gy; source to distance 10 $\mathrm{cm}$; at the rate of 20.16 Gy per minute; irradiation at CRIJAF, Nilganj, West Bengal). Control and treated seeds were sown (50 seeds in each lot) in the experimental field of Kalyani University (spacing of $15 \mathrm{~cm}$ between plants and $40 \mathrm{~cm}$ between lines) to raise $M_{1}$ and subsequently $M_{2}$ generation (plant to row) during rain fed seasons of 2005 and 2006 respectively. Macromutants were scored at $M_{2}$. Selfed $M_{2}$ plant types were used to raise $\mathrm{M}_{3}$ and subsequently $\mathrm{M}_{4}$ generations.

Several leaf and flower mutants recovered at $\mathrm{M}_{2}$ were compared with their respective control and ' $\mathrm{t}$ ' test was performed whenever needed. Crossings were made between normal ( as female parent) and mutants (narrow leaf, elongated leaf, thick leaf, ovate leaf, ternate and long petiole; male parent) and $F_{1}$ and $F_{2}$ plants were raised. The $F_{2}$ were used for estimating the segregating ratio of different leaf traits using the Chi-square $\left(\alpha^{2}\right)$ test segregation patterns of white flower and pigmented flower mutants (colours confirmed by Horticultural Colour Chart I and II) were studied from selfed $\mathrm{M}_{2}$ mutants grown in $M_{3}$ generation and the segregating ratios were confirmed by $\mathrm{x}^{2}$-test.

Meiotic analysis (PMC and pollen grains were stained in $2 \%$ aceto - carmine solution) was performed in control and mutant plant types (flower buds fixed from 2 to 3 plants of each category in 1:3 (v/v) acetic alcohol and preserved in $70 \%$ alcohol) from $M_{2}$ and $M_{3}$ plants. Photomicrographs were taken from temporary squash preparations.

Control and mutant types were compared for 10 different agronomic parameters (Table 1 ) by growing them in randomized block design at $\mathrm{M}_{4}$ both at irrigated (January to April) and rain fed kharif (May to August) seasons 2008 with three replications. Plot size was $3 \mathrm{~m} \mathrm{X}$ $1.5 \mathrm{~m}$ with 4 rows in each plot and each row was $250 \mathrm{~cm}$ long. Spacing was $30 \mathrm{~cm}$ between rows and $10 \mathrm{~cm}$ between plants. Five plants were randomly taken from each replication and a total of 15 plants were assessed for each plant type and the data obtained were statistically analyzed.

Seed protein was extracted following Osborne (1962) and estimated as per Lowry et. al. (1951). Oil was extracted in petroleum ether $\left(60-80^{\circ} \mathrm{C}\right.$ b. p. for 5 hours) by soxhlet apparatus from seeds dried in the sun (2 consecutive days, 5 hours).

Results and discussion

Morphology. Narrow leaf (narrow, oblong to lanceolate, entire margin; length: mutant $-15.5 \mathrm{~cm} \pm 0.41$, control $13.7 \mathrm{~cm} \pm 0.19, \mathrm{t}=1.68, \mathrm{df}=28, \mathrm{p}>0.05$; breadth: mutant -
Research article

CIndian Society for Education and Environment (iSee)
"Cytogenetics of sesame mutants" http://www.indjst.org
Chowdhury et al. Indian J.Sci.Technol. 


\begin{tabular}{|c|c|c|c|c|c|c|c|c|c|c|c|}
\hline \multirow[t]{2}{*}{ Plant types } & \multicolumn{11}{|c|}{ Attributes } \\
\hline & & $\begin{array}{c}\text { Plant } \\
\text { height }(\mathrm{cm})\end{array}$ & $\begin{array}{c}\text { No of } \\
\text { primary } \\
\text { branche } \\
\text { s/ plant }\end{array}$ & $\begin{array}{c}\text { Total } \\
\text { branche } \\
\text { s/ plant }\end{array}$ & $\begin{array}{l}\text { Distance } \\
\text { from } \\
\text { base to I } \\
\text { branch } \\
(\mathrm{cm})\end{array}$ & $\begin{array}{c}\text { Capsule } \\
\text { on the } \\
\text { main } \\
\text { axis }\end{array}$ & $\begin{array}{c}\text { Total } \\
\text { capsule/ } \\
\text { plant }\end{array}$ & $\begin{array}{c}\text { Capsule } \\
\text { length } \\
(\mathrm{cm})\end{array}$ & $\begin{array}{l}\text { Seed } \\
\text { yield/ } \\
\text { plant } \\
(\mathrm{gm})\end{array}$ & $\begin{array}{c}\text { Seed } \\
\text { protein } \\
\text { content } \\
(\%)\end{array}$ & $\begin{array}{c}\text { Seed fatty } \\
\text { oil content } \\
(\%)\end{array}$ \\
\hline Control & $\begin{array}{l}\mathrm{K} \\
\mathrm{I}\end{array}$ & $\begin{array}{l}113.4 \pm 1.3 \\
110.3 \pm 1.4\end{array}$ & $\begin{array}{l}5.9 \pm 0.3 \\
4.8 \pm 0.2\end{array}$ & $\begin{array}{l}9.1 \pm 0.1 \\
9.1 \pm 0.2\end{array}$ & $\begin{array}{l}25.5 \pm 2.7 \\
25.8 \pm 0.2\end{array}$ & $\begin{array}{l}22.2 \pm 1.2 \\
25.3 \pm 1.3\end{array}$ & $\begin{array}{l}62.7 \pm 1.5 \\
57.1 \pm 1.1\end{array}$ & $\begin{array}{l}2.2 \pm 0.02 \\
2.3 \pm 0.01\end{array}$ & $\begin{array}{l}6.6 \pm 0.03 \\
6.3 \pm 0.1\end{array}$ & $\begin{array}{l}12.8 \pm 0.2 \\
12.6 \pm 0.5\end{array}$ & $\begin{array}{l}34.4 \pm 0.8 \\
35.2 \pm 0.8\end{array}$ \\
\hline $\begin{array}{l}\text { Narrow } \\
\text { leaf }\end{array}$ & $\begin{array}{l}\mathrm{K} \\
\mathrm{I}\end{array}$ & $\begin{array}{l}60.0 \pm 1.3 \\
54.9 \pm 0.6\end{array}$ & $\begin{array}{l}2.1 \pm 0.1 \\
2.5 \pm 0.2\end{array}$ & $\begin{array}{l}3.3 \pm 0.1 \\
4.1 \pm 0.4\end{array}$ & $\begin{array}{l}21.3 \pm 0.7 \\
19.2 \pm 0.4\end{array}$ & $\begin{array}{l}29.6 \pm 1.9 \\
23.9 \pm 2.8\end{array}$ & $\begin{array}{l}51.7 \pm 1.1 \\
38.1 \pm 2.5\end{array}$ & $\begin{array}{l}2.1 \pm 0.01 \\
2.0 \pm 0.01\end{array}$ & $\begin{array}{l}7.2 \pm 0.2 \\
5.7 \pm 0.3\end{array}$ & $\begin{array}{l}17.3 \pm 0.6 \\
17.4 \pm 0.4\end{array}$ & $\begin{array}{l}30.5 \pm 0.3 \\
30.7 \pm 1.7\end{array}$ \\
\hline $\begin{array}{l}\text { Elongated } \\
\text { leaf }\end{array}$ & $\begin{array}{l}\mathrm{K} \\
\mathrm{I}\end{array}$ & $\begin{array}{l}83.2 \pm 0.3 \\
84.5 \pm 1.0\end{array}$ & $\begin{array}{l}2.3 \pm 0.2 \\
2.3 \pm 0.1\end{array}$ & $\begin{array}{l}5.0 \pm 0.1 \\
4.1 \pm 0.1\end{array}$ & $\begin{array}{l}25.1 \pm 1.5 \\
26.0 \pm 0.9\end{array}$ & $\begin{array}{l}21.9 \pm 0.6 \\
22.1 \pm 0.4\end{array}$ & $\begin{array}{l}48.1 \pm 6.2 \\
43.3 \pm 0.8\end{array}$ & $\begin{array}{l}2.3 \pm 0.01 \\
2.2 \pm 0.02\end{array}$ & $\begin{array}{l}6.8 \pm 0.6 \\
6.2 \pm 0.1\end{array}$ & $\begin{array}{l}16.6 \pm 0.3 \\
16.1 \pm 0.3\end{array}$ & $\begin{array}{l}33.4 \pm 1.7 \\
32.5 \pm 1.0\end{array}$ \\
\hline Thick leaf & $\begin{array}{l}\mathrm{K} \\
\mathrm{I}\end{array}$ & $\begin{array}{l}185.7 \pm 2.7 \\
178.0 \pm 1.7\end{array}$ & $\begin{array}{l}6.4 \pm 0.2 \\
6.1 \pm 0.2\end{array}$ & $\begin{array}{l}12.8 \pm 0.4 \\
11.7 \pm 0.2\end{array}$ & $\begin{array}{l}69.1 \pm 1.3 \\
68.3 \pm 1.3\end{array}$ & $\begin{array}{l}41.1 \pm 0.6 \\
33.0 \pm 0.9\end{array}$ & $\begin{array}{l}82.6 \pm 1.9 \\
79.1 \pm 1.7\end{array}$ & $\begin{array}{l}2.3 \pm 0.01 \\
2.3 \pm 0.01\end{array}$ & $\begin{array}{l}7.9 \pm 0.1 \\
7.7 \pm 0.1\end{array}$ & $\begin{array}{l}21.4 \pm 0.6 \\
20.1 \pm 0.7\end{array}$ & $\begin{array}{l}27.6 \pm 0.4 \\
30.4 \pm 0.5\end{array}$ \\
\hline Ovate leaf & $\begin{array}{l}\mathrm{K} \\
\mathrm{I}\end{array}$ & $\begin{array}{l}67.2 \pm 1.5 \\
67.1 \pm 0.4\end{array}$ & $\begin{array}{l}2.4 \pm 0.1 \\
2.6 \pm 0.1\end{array}$ & $\begin{array}{l}5.1 \pm 0.1 \\
4.9 \pm 0.1\end{array}$ & $\begin{array}{l}20.6 \pm 0.3 \\
20.3 \pm 0.6\end{array}$ & $\begin{array}{l}13.9 \pm 0.3 \\
13.9 \pm 0.2\end{array}$ & $\begin{array}{l}29.9 \pm 0.2 \\
30.7 \pm 0.2\end{array}$ & $\begin{array}{l}2.3 \pm 0.01 \\
2.3 \pm 0.01\end{array}$ & $\begin{array}{l}5.3 \pm 0.1 \\
5.3 \pm 0.1\end{array}$ & $\begin{array}{l}15.4 \pm 0.6 \\
14.8 \pm 0.3\end{array}$ & $\begin{array}{l}32.9 \pm 0.8 \\
32.5 \pm 1.8\end{array}$ \\
\hline Ternate & $\begin{array}{l}\mathrm{K} \\
\mathrm{I}\end{array}$ & $\begin{array}{l}74.3 \pm 1.0 \\
71.2 \pm 0.6\end{array}$ & $\begin{array}{l}2.9 \pm 0.1 \\
2.9 \pm 0.1\end{array}$ & $\begin{array}{l}4.1 \pm 0.1 \\
4.3 \pm 0.2\end{array}$ & $\begin{array}{l}21.7 \pm 0.2 \\
20.4 \pm 0.3\end{array}$ & $\begin{array}{l}24.0 \pm 0.6 \\
22.7 \pm 0.7\end{array}$ & $\begin{array}{l}53.8 \pm 1.9 \\
53.0 \pm 1.2\end{array}$ & $\begin{array}{l}2.1 \pm 0.01 \\
2.1 \pm 0.02\end{array}$ & $\begin{array}{l}6.6 \pm 0.1 \\
6.7 \pm 0.1\end{array}$ & $\begin{array}{l}13.2 \pm 0.4 \\
13.0 \pm 0.5\end{array}$ & $\begin{array}{l}32.3 \pm 0.4 \\
32.4 \pm 0.5\end{array}$ \\
\hline $\begin{array}{l}\text { Long } \\
\text { petiole }\end{array}$ & $\begin{array}{l}\mathrm{K} \\
\mathrm{I}\end{array}$ & $\begin{array}{l}85.5 \pm 0.8 \\
82.2 \pm 0.3\end{array}$ & $\begin{array}{l}2.9 \pm 0.1 \\
2.8 \pm 0.1\end{array}$ & $\begin{array}{l}4.6 \pm 0.2 \\
4.6 \pm 0.2\end{array}$ & $\begin{array}{l}26.8 \pm 0.9 \\
28.1 \pm 1.0\end{array}$ & $\begin{array}{l}19.9 \pm 0.4 \\
20.4 \pm 0.9\end{array}$ & $\begin{array}{l}39.9 \pm 1.0 \\
41.8 \pm 1.4\end{array}$ & $\begin{array}{l}1.2 \pm 0.01 \\
1.2 \pm 0.01\end{array}$ & $\begin{array}{l}4.0 \pm 0.1 \\
4.2 \pm 0.1\end{array}$ & $\begin{array}{l}14.9 \pm 0.2 \\
13.9 \pm 0.2\end{array}$ & $\begin{array}{l}33.4 \pm 0.5 \\
33.8 \pm 1.2\end{array}$ \\
\hline $\begin{array}{l}\text { White } \\
\text { flower }\end{array}$ & $\begin{array}{l}\mathrm{K} \\
\mathrm{I}\end{array}$ & $\begin{array}{l}67.4 \pm 0.6 \\
64.8 \pm 1.8\end{array}$ & $\begin{array}{l}3.4 \pm 0.4 \\
3.3 \pm 0.2\end{array}$ & $\begin{array}{l}7.5 \pm 0.3 \\
7.2 \pm 0.2\end{array}$ & $\begin{array}{l}18.6 \pm 0.3 \\
19.6 \pm 0.2\end{array}$ & $\begin{array}{l}19.5 \pm 0.4 \\
17.9 \pm 1.0\end{array}$ & $\begin{array}{l}46.8 \pm 0.3 \\
40.3 \pm 2.5\end{array}$ & $\begin{array}{l}2.1 \pm 0.01 \\
2.1 \pm 0.01\end{array}$ & $\begin{array}{l}3.6 \pm 0.1 \\
3.8 \pm 0.2\end{array}$ & $\begin{array}{l}15.6 \pm 0.4 \\
15.3 \pm 0.4\end{array}$ & $\begin{array}{l}37.4 \pm 0.4 \\
35.9 \pm 0.4\end{array}$ \\
\hline $\begin{array}{l}\text { Pigmented } \\
\text { flower }\end{array}$ & $\begin{array}{l}\mathrm{K} \\
\mathrm{I}\end{array}$ & $\begin{array}{l}98.3 \pm 2.7 \\
94.2 \pm 3.1\end{array}$ & $\begin{array}{l}4.0 \pm 0.1 \\
2.9 \pm 0.3\end{array}$ & $\begin{array}{l}6.0 \pm 0.2 \\
5.0 \pm 0.6\end{array}$ & $\begin{array}{l}34.1 \pm 3.8 \\
28.2 \pm 1.3\end{array}$ & $\begin{array}{l}23.6 \pm 2.7 \\
20.9 \pm 2.2\end{array}$ & $\begin{array}{l}44.2 \pm 3.2 \\
42.6 \pm 2.8\end{array}$ & $\begin{array}{l}2.3 \pm 0.05 \\
2.5 \pm 0.01\end{array}$ & $\begin{array}{l}6.0 \pm 0.5 \\
5.8 \pm 0.1\end{array}$ & $\begin{array}{l}15.0 \pm 0.6 \\
15.0 \pm 0.7\end{array}$ & $\begin{array}{l}37.6 \pm 1.3 \\
37.7 \pm 2.2\end{array}$ \\
\hline $\begin{array}{l}\text { CD at 5\% } \\
\text { level }\end{array}$ & $\begin{array}{l}\mathrm{K} \\
\mathrm{l}\end{array}$ & $\begin{array}{l}6.83 \\
5.59\end{array}$ & $\begin{array}{l}0.91 \\
0.41\end{array}$ & $\begin{array}{l}0.92 \\
0.66\end{array}$ & $\begin{array}{l}3.82 \\
2.46\end{array}$ & $\begin{array}{l}3.02 \\
2.87\end{array}$ & $\begin{array}{l}5.36 \\
4.24\end{array}$ & $\begin{array}{l}0.14 \\
0.12\end{array}$ & $\begin{array}{l}0.66 \\
0.42\end{array}$ & $\begin{array}{l}1.15 \\
2.26\end{array}$ & $\begin{array}{l}1.87 \\
2.48\end{array}$ \\
\hline
\end{tabular}

\section{I = irrigated; $K=$ kharif}

$6.13 \mathrm{~cm} \pm 0.15$, control $-11.06 \mathrm{~cm} \pm 0.27, \mathrm{t}=7.62, \mathrm{df}=28$, p<0.001, area: mutant -42.67 sq. $\mathrm{cm} \pm 2.74$, control -87.2 sq.cm. $\pm 4.19, t=13.74, d f=28, p<0.001$ ), elongated leaf (length $-17.83 \mathrm{~cm} \pm 0.14$, breadth $-11.1 \mathrm{~cm} \pm 0.48$, area $106.67 \mathrm{sq} \mathrm{cm} \pm 4.25$ ), thick leaf (leaves were leathery), ovate leaf (serrated margins, hairy on upper surface, short and thick petiole), ternate (three leaves per node and each leaf axil bearing three fruits, altered phyllotaxy opposite decussate to whorled), long petiole (length $19.0 \mathrm{~cm}$ to $22.0 \mathrm{~cm}$ in mutant compared to $7.0 \mathrm{~cm}$ to 9.0 $\mathrm{cm}$ in control; petioles more or less right angles to main axis), white flower (corolla tube and flap white [8/2], phlox purple [632 to 632/3] colour in flaps and white colour corolla tubes in control) and pigmented flower (mutant corolla tube - phlox purple [632/3], flap - phlox purple $\left[632 /{ }_{2}\right]$ ) mutant plant types were spotted at $M_{2}$ (Figs. 1-9) in different doses of $\mathrm{x}$-ray and gamma- ray irradiations (Chowdhury \& Datta, 2008). Mary and Jayabalan (1995) induced mutation affecting leaf morphology in sesame at $M_{2}$ following EMS treatments to seeds. Sengupta and Datta (2005) identified a narrow leaf mutant in sesame following nitrous acid and hydrogen peroxide treatments in different doses, and the mutant yielded higher number of capsule/plant on the main axis than control.

Inheritance of traits: $\mathrm{F}_{1}$ 's raised from different set of crossings made between normal and mutants were of normal phenotypes. $\mathrm{F}_{2}$ segregation revealed that narrow leaf (normal 33, mutant 12 , total $45, \kappa^{2}=0.067$ for $3: 1$ at $1 \mathrm{df}, \mathrm{p}>0.75$ ), elongated leaf (normal 44 , mutant 12 , total $56, \mathrm{x}^{2}=0.423$ for 3 : 1 at $1 \mathrm{df}, \mathrm{p}>0.50$ ), thick leaf (normal 86 , mutant 27 , total $113, \mathrm{k}^{2}=0.073$ for 3 : 1 at $1 \mathrm{df}, \mathrm{p}>$
0.75 ), ovate leaf (normal 35 , mutant 11 , total $46, \mathrm{x}^{2}=$ 0.029 for $3: 1$ at $1 \mathrm{df}, \mathrm{p}>0.80$ ), ternate (normal 27 , mutant 08 , total $35, \mathrm{k}^{2}=0.085$ for 3 : 1 at $1 \mathrm{df}, \mathrm{p}>0.75$ ), long petiole (control 62 , mutant 19 , total $81, \alpha^{2}=0.103$ for 3 : 1 at $1 \mathrm{df}, \mathrm{p}>0.70)$ traits were monogenic recessive to normal trait(s). $\mathrm{M}_{3}$ segregation of white flower (normal 08 , mutant 03 , total $11, \mathrm{\kappa}^{2}=0.029$ for 3 : 1 at $1 \mathrm{df}, \mathrm{p}<0.90$ ) and pigmented flower (normal 21, mutant 06, total 27, $\mathrm{k}^{2}$ $=0.111$ for $3: 1$ at $1 \mathrm{df}, \mathrm{p}>0.70$ ) also suggested possible monogenic recessive inheritance of the flower colour traits. Most mutations in angiosperm are reported to be controlled by single pair of recessive alleles (Brock, 1971; Gaur \& Gour, 1999).

Meiosis: Meiotic analysis revealed $2 \mathrm{n}=26$ chromosomes always and the chromosomes formed bivalents (control: 12.93 /cell; mutant: $12.26 /$ cell to $12.93 /$ cell) and univalents (control: 0.14 / cell; mutant 0.14 / cell to 1.48 / cell). Among the mutants, univalent frequency was higher in long petiole (1.48/ cell). Bivalents and univalents tended to form variable groups (3 to 10) in control (57.74\% meiocytes) and in mutant plant types ( $46.81 \%$ to $65.20 \%$ ). Predominant group class noted among the plant types was 8 (control: $28.17 \%$ cells, mutant: $17.02 \%$ to $47.62 \%$ cells) and it was in conformity to earlier findings of Sengupta and Datta (2003). Predominant chromosomal association noted among the plant types was 13 II (control: 92.96\%; mutants: $50.00 \%$ to $95.88 \%$ ). Average chromosome associations per cell at metaphase I of the plant types were recorded to be $12.93 \mathrm{II}+0.14 \mathrm{I}$ in control, $12.89 \mathrm{II}+0.21$ I in narrow leaf, $12.93 \mathrm{II}+0.14 \mathrm{I}$ in elongated leaf and thick leaf, $12.90 \mathrm{II}+0.19 \mathrm{I}$ in ovate
Research article

CIndian Society for Education and Environment (iSee)
"Cytogenetics of sesame mutants" http://www.indjst.org
Chowdhury et al. Indian J.Sci.Technol. 
leaf, $12.87 \mathrm{II}+0.27$ I in ternate, $12.26 \mathrm{II}+$ $1.48 \mathrm{I}$ in long petiole, $12.92 \mathrm{II}+0.16 \mathrm{I}$ in white flower and $12.91 \mathrm{II}+0.18 \mathrm{I}$ in pigmented flower. Compared to $100.00 \%$ cells with equal (13/13) anaphase I separation in control, the mutants had $98.80 \%$ to $100.00 \%$ cells. Pollen fertility studied in control was $83.50 \%$ and it varied from $38.90 \%$ (white flower) to $80.20 \%$ (ternate). Pollen fertility and $\mathrm{Al}$ chromosome separation was noncorrelated $(r=0.34$, df $8, p>0.05)$. Analysis of cytogenetical parameters indicated that the mutants were possibly the outcome of genetical causes rather than cytological disturbances.

Agronomic traits: Results indicated (Table 1) that irrespective of seasons thick leaf mutant was most desirable plant type and found to possess superior agronomic traits like plant height, primary and total branches per plant, capsule on main axis, distance from base to first branching, total capsule per plant, seed yield and seed protein content than control. Compared to control, seed protein content also enhanced in narrow leaf, elongated leaf, white flower and pigmented flower mutants; while pigmented flower mutant plants were with increased seed fatty oil content. Capsule length also increased in pigmented flower mutant than control. Thus, these mutants correspond closely to the ideotype being looked for in the species and offer scope of their direct selection; while ovate leaf, ternate and long petiole plant types may
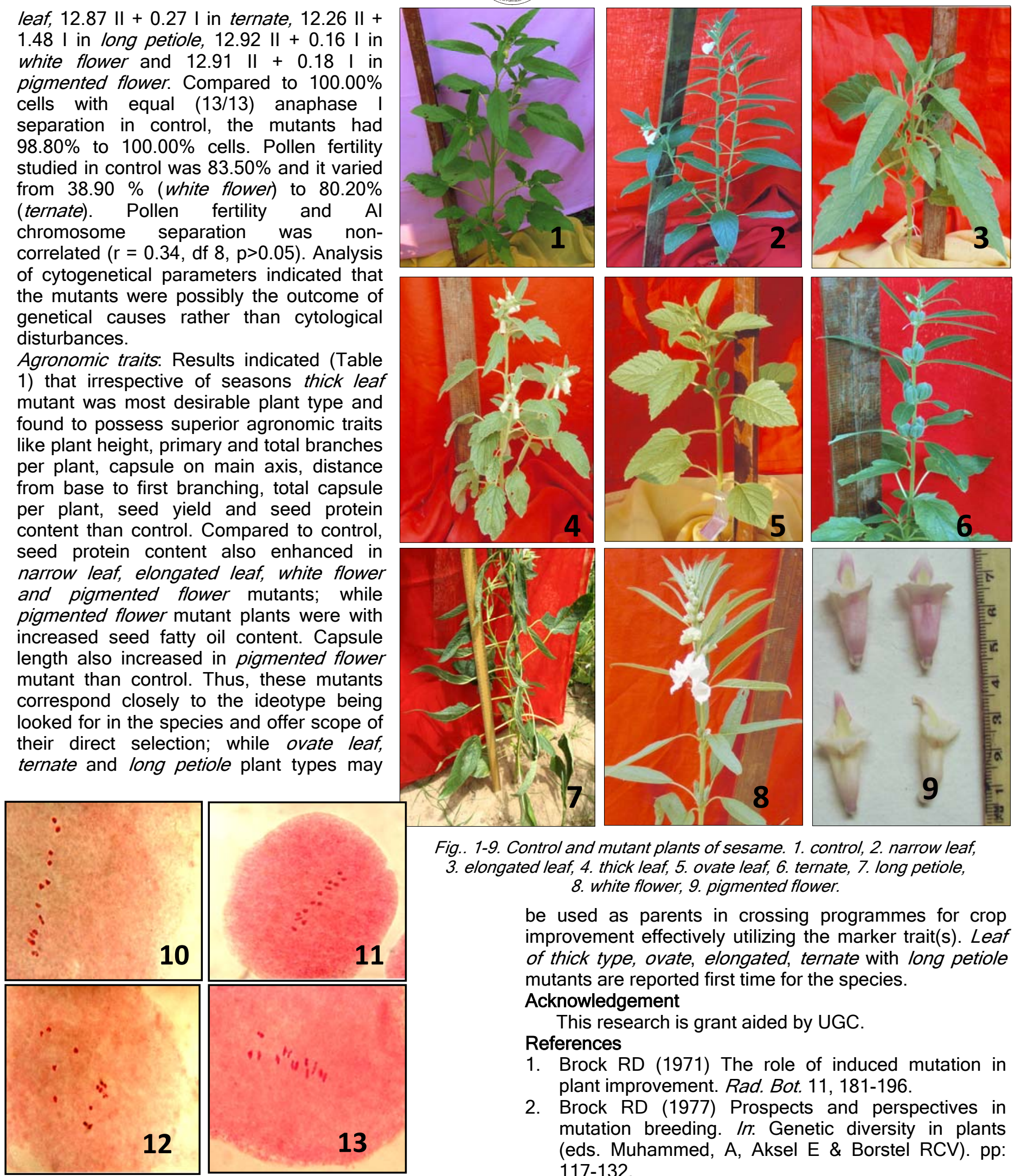

Fig.. 1-9. Control and mutant plants of sesame. 1. control, 2. narrow leaf, 3. elongated leaf, 4. thick leaf, 5. ovate leaf, 6. ternate, 7. long petiole, 8. white flower, 9. pigmented flower.

be used as parents in crossing programmes for crop improvement effectively utilizing the marker trait(s). Leaf of thick type, ovate, elongated, ternate with long petiole mutants are reported first time for the species.

Acknowledgement

This research is grant aided by UGC.

\section{References}

1. Brock RD (1971) The role of induced mutation in plant improvement. Rad. Bot. 11, 181-196.

2. Brock RD (1977) Prospects and perspectives in mutation breeding. I $n$ : Genetic diversity in plants (eds. Muhammed, A, Aksel E \& Borstel RCV). pp: 117-132.

Figs. 10-13. Meiotic metaphase I in mutants $(2 n=26)$. 10. 13II-3II(1)+2II(3)+1II(3)7 groups, 11. 13II - 3II(2) $+2 I I(2)+1 I I(3)-7$ group class, 12. 13II -2II(4)+1II(5) -9 groups, 13. 13II-2II(5)+1II(3) - 8 groups.

Research article

CIndian Society for Education and Environment (iSee)
"Cytogenetics of sesame mutants" http://www.indjst.org
Chowdhury et al. Indian J.Sci.Technol. 
4. Datta AK and Biswas AK (1985) Induced mutagenesis in Nigella sativa L. Cytologia. $50,545-$ 562

5. Datta AK and Rang SK (2001) A viable chlorophyll mutant in black cumin (Nigella sativa L.). Indian J. Genet. 61, 293-294.

6. Gaul H (1964) Mutations in plant breeding. Rad. Bot. 4, 155-232.

7. Gaur P and Gour VK (1999) An induced fasciated mutant of Chickpea (Cicer arietinum L.). Indian J. Genet. 59, 325- 330.

8. Iqbal M and Datta A K (2007) Induced mutagenesis in Withania somnifera. J. Trop. Med. Plants. 8, 47-53.

9. Kharkwal MC (2000) Induced mutation in chick pea (Cicer arietinum L.). V. Evaluation of micromutations. Indian J. Genet. 61, 115-124.

10. Lowry $\mathrm{OH}$, Rosebrough $\mathrm{NJ}$, Farr $\mathrm{AL}$ and Randell RJ (1951) Protein measurement with Folin Phenol reagent. J. Biol. Chem. 193, 265-275.

11. Mary RJ and Jayabalan N (1995) EMS induced variability in sesame. Crop Improv. 22, 170-174.

12. Mukherjee $M$ and Datta AK (2006) Induced viable macromutants in (sweet basil) Ocimum basilicum. J. Trop. Med. Plants. 7, 123-128.

13. Osborn DJ (1962) Effects of kinetin on protein and nucleic acid metabolism in Xanthium leaves during senescence. Plant. Physiol. 87, 595-602.

14. Sengupta K and Chatterjee $D$ (1982) A review of genetical and breeding work in different oil seed crops at pulses and Oil Seed Research Station, West Bengal. Pulses and Oilseeds in West Bengal. Govt. of West Bengal. pp: 149-158.

15. Sengupta $S$ and Datta AK (2003) Secondary chromosome associations in control and mutant plant types of Sesamum indicum L. Cytologia. 68, 141-145.

16. Sengupta S and Datta AK (2005) Induced narrow leaf mutant of sesame (Sesamum indicum L.). Indian J. Genet. 65, 59-60. 